\title{
Computing Zernike polynomials of arbitrary degree using the discrete Fourier transform
}

\author{
Augustus J. E. M. Janssen \\ a.j.e.m.janssen@philips.com
}

Peter Dirksen

peter.dirksen@philips.com

\author{
Philips Research Europe, HTC 36 \\ Philips Research Europe, HTC 4
}

The conventional representation of Zernike polynomials $R_{n}^{m}(\rho)$ gives unacceptable numerical results for large values of the degree $n$. We present an algorithm for the computation of Zernike polynomials of arbitrary degree $n$. The algorithm has the form of a discrete Fourier (cosine) transform which comes with advantages over other methods in terms of computation time, accuracy and ease of implementation. As an application we consider the effect of NA-scaling on the lower-order aberrations of an optical system in the presence of a very high order aberration. [DOI: 10.2971/jeos.2007.07012]

Keywords: Zernike polynomial, aberration, high-order, discrete Fourier transform, NA-scaling

\section{INTRODUCTION}

The (radial part of the) Zernike polynomials $R_{n}^{m}(\rho)$ are widely used in the representation of the aberrations of optical systems and in the computation of the diffraction integral defining the point-spread function of these systems [1]-[5].When we are dealing with smooth exit pupil functions, it is, in general, sufficient to consider the $R_{n}^{m}$ for modest values of the degree $n$ and azimuthal order $m$, say, $n+m \leq 12$. For such pupil functions, the conventional polynomial representation [1]

$$
\begin{aligned}
& R_{n}^{m}(\rho)= \\
& =\sum_{s=0}^{(n-m) / 2} \frac{(n-s) !(-1)^{s}}{\left(\frac{n-m}{2}-s\right) !\left(\frac{n+m}{2}-s\right) ! s !} \rho^{n-2 s}, 0 \leq \rho \leq 1,
\end{aligned}
$$

can be used to calculate the Zernike polynomials. Some low order Zernike polynomials are shown in Table 1.

\begin{tabular}{|c|c|c|}
\hline Degree $n$ & $m$ & $R_{n}^{m}(\rho)$ \\
\hline 0 & 0 & 1 \\
\hline 1 & 1 & $\rho$ \\
\hline 2 & 0 & $2 \rho^{2}-1$ \\
\hline 2 & 2 & $\rho^{2}$ \\
\hline 4 & 0 & $6 \rho^{4}-6 \rho^{2}+1$ \\
\hline 3 & 1 & $3 \rho^{3}-2 \rho$ \\
\hline 3 & 3 & $\rho^{3}$ \\
\hline
\end{tabular}

TABLE 1 Low order Zernike polynomials

In the case that the exit pupil function contains discontinuities, or is roughly behaved in a more general sense, it is necessary to consider Zernike polynomials of much higher degree and order. For instance, when the pupil function has a central obstruction ( 0 and 1 on two concentric sets $0 \leq \rho<a$ and $a \leq \rho \leq 1)$, the coefficient of $R_{n}^{0}(\rho)$ in the Zernike expansion of the pupil function can be shown to decay only like $n^{-1 / 2}$. Then Eq. (1) becomes cumbersome because of the high-order factorials that are required. Also, for $m=0$, it can be shown from the Stirling's formula that the largest coefficient of $\rho^{n-2 s}$ occurring in the series in Eq. (1) behaves like $(1+\sqrt{2})^{n}$. Accordingly, when computing with $d$ digits, Eq. (1) produces errors of the order of unity or larger from $n=d / \log (1+\sqrt{2})$ onwards. Hence, for the commonly used 15 digits precision, one has serious problems from $n=40$ onwards, as shown in Figure 1, top, right. An alternative to compute Zernike polynomials is to use recursions for them such as those found in Ref. [6]. These recursion schemes are, however, computationally more expensive and less direct than a formula like Eq. (1) and their accuracy due to error propagation is also an issue.

In this paper, we present a new computation scheme in which one can allow degrees as large as $10^{5}$ without problems. This new algorithm is of the discrete-cosine transform (DCT) type, and is direct and transparent. Furthermore, the computation can be done using the FFT-algorithm which comes with the following advantages [7, 8]:

- Very favorable and well-established accuracy

- Simultaneous computation of all Zernike polynomials of the same degree $n$ in as few as $\mathrm{O}(n \log n)$ operations

As an application we consider the effect of NA-scaling on the lower-order aberrations of an optical system in the presence of a very high order aberration. For this we use a recently found 
formula [9], entirely in terms of Zernike polynomials, for the Zernike coefficients of scaled pupils.

\section{DCT FORMULA FOR ZERNIKE POLYNOMIALS}

In Appendix A we show that

$$
R_{n}^{m}(\rho)=\frac{1}{N} \sum_{k=0}^{N-1} U_{n}\left(\rho \cos \frac{2 \pi k}{N}\right) \cos \frac{2 \pi m k}{N}, \quad 0 \leq \rho \leq 1,
$$

where $N$ is any integer $>n+m$. In Eq. (2) we have integer $n, m \geq 0$ with $n-m$ even and $\geq 0$ (as usual), and $U_{n}$ is the Chebyshev polynomial of the second kind and of degree $n$. No matter how large $n$ is, the evaluation of $U_{n}(x)$ is no problem since we have

$$
U_{n}(x)=\frac{\sin (n+1) v}{\sin v}, \quad x=\cos v .
$$

Eq. (2) is a consequence of the formula (A.10) that represents $R_{n}^{m}(\rho)$ as an integral of a trigonometric polynomial of degree $n+m$ over a periodicity interval. Such an integral can be computed error-free as a series when the number of equidistant sample points $N$ exceeds the degree $n+m$. It also follows from this that the right-hand side of Eq. (2) (and that of Eq. (A.10)) vanish when $m>n$ or when $n$ and $m$ have different parity (with again $N>n+m$ ).

Eq. (2) gives $R_{n}^{m}(\rho)$ as the $m^{\text {th }}$ component of the DCT of the sequence $\left(U_{n}(\rho \cos 2 \pi k / N)\right)_{k=0,1, \ldots, N-1}$, hence we get all $R_{n}^{m}(\rho)$, with $m \geq 0$ and $m=n, n-2, \ldots$, using $O(N \log N)$ operations. Since $m \leq n$, it is sufficient to take $N$ any integer $>2 n$.

In Figure 1, top, we show $R_{n}^{m}(\rho)$ as a function of $\rho, 0 \leq \rho \leq 1$, computed according to Eq. (1) and Eq. (2), using 16 decimal places, for $m=17, n=39$ and for $m=0, n=50$. We see that Eq. (1) gives unacceptable results for the case $m=0, n=50$ from $\rho=0.8$ onwards.

In Figure 1, bottom, we show $R_{n}^{m}(\rho)$, computed according to Eq. (2), with $m=0$ and $n=10000$ and $\rho$ very close to 1 . We see that $R_{n}^{m}(\rho=1)=1$ which is in agreement with the theory [1].

\section{AN APPLICATION: HIGH-ORDER ABERRATIONS AND SCALING}

In lithographic imaging systems, the numerical aperture (NA) is varied intentionally below its maximum value so as to optimize the performance for the particular object to be imaged. In Ref. [9] the effect of NA-scaling on the Zernike coefficients describing the optical system has been concisely expressed in terms of Zernike polynomials. Thus we consider a pupil function

$$
P(\rho, \vartheta)=\exp \{i \Phi(\rho, \vartheta)\}, \quad 0 \leq \rho \leq 1,0 \leq \vartheta \leq 2 \pi,
$$

in polar coordinates with real phase $\Phi$, and we assume that $\Phi$ is expanded as a Zernike series according to

$$
\Phi(\rho, \vartheta)=\sum_{n, m} \alpha_{n}^{m} R_{n}^{m}(\rho) \cos m \vartheta
$$
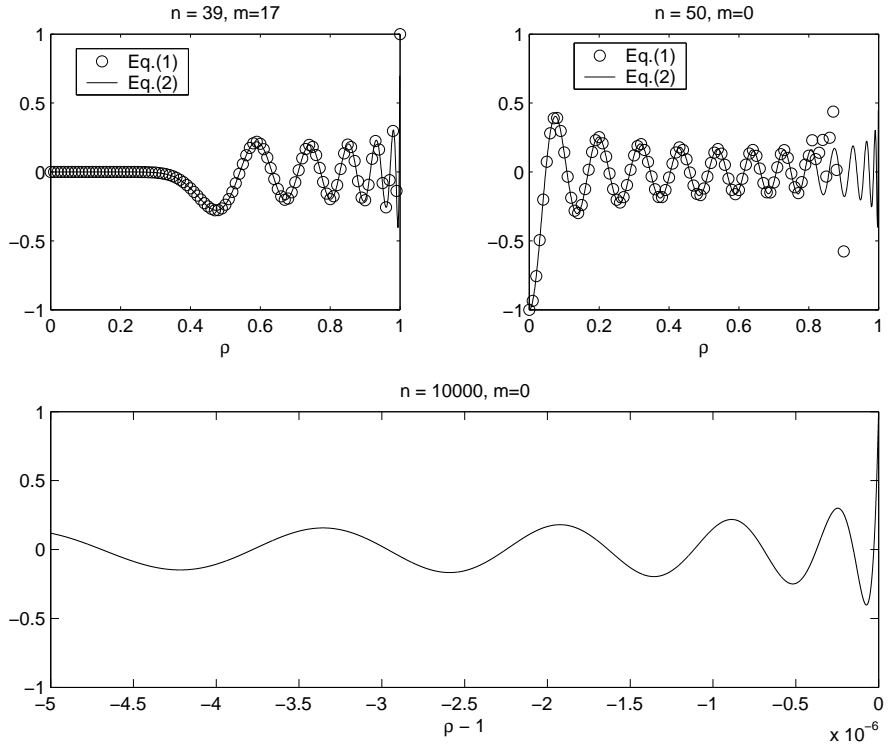

FIG. 1 Top: $R_{n}^{m}(\rho)$ as a function of $\rho, 0 \leq \rho \leq 1$, computed according to Eq. (1) and Eq. (2), using 16 digits, for $m=17, n=39$ and for $m=0, n=50$. Bottom: $R_{n}^{m}(\rho)$, computed according to Eq. (2), with $m=0$ and $n=10000$ and $\rho$ very close to 1

Scaling to a pupil with relative size $\varepsilon=\mathrm{NA} / \mathrm{NA}_{\max } \leq 1$ requires computation of the Zernike coefficients $\alpha_{n}^{m}(\varepsilon)$ of the scaled phase function $\Phi(\varepsilon \rho, \vartheta)$. For $m=0,1, \ldots$ the $\alpha_{n}^{m}(\varepsilon)$ are given in terms of the $\alpha_{n}^{m}$ as

$$
\alpha_{n}^{m}(\varepsilon)=\sum_{n^{\prime}} \alpha_{n^{\prime}}^{m}\left[R_{n^{\prime}}^{n}(\varepsilon)-R_{n^{\prime}}^{n+2}(\varepsilon)\right], \quad n=m, m+2, \ldots,
$$

where the summation is over $n^{\prime}=n, n+2, \ldots\left(R_{n}^{n+2} \equiv 0\right)$. In case of a non-smooth phase function $\Phi$, one should expect significant values of $\alpha_{n^{\prime}}^{m}$ for very high degrees $n^{\prime}$. Also, scaling is normally done using values of $\varepsilon$ close to its maximum 1 , where Eq. (1) produces the largest numerical error. Thus, formula (6) is not practicable in these cases when Eq. (1) is used to evaluate $R_{n^{\prime}}^{n}(\varepsilon)-R_{n^{\prime}}^{n+2}(\varepsilon)$, but becomes so when Eq. (2) is used instead.
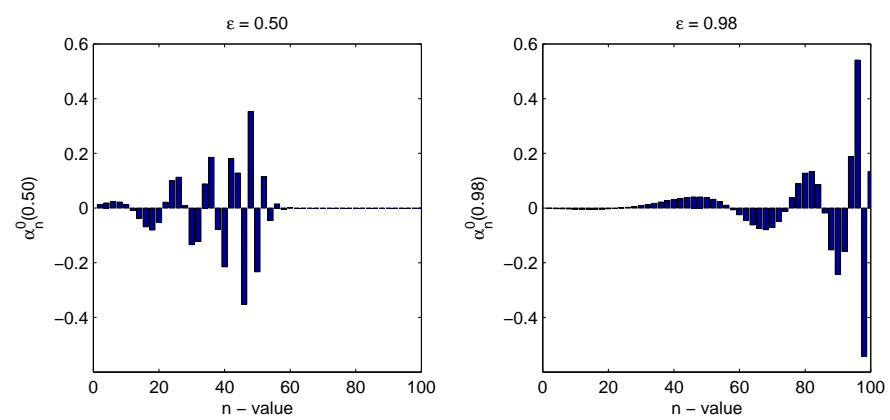

FIG. 2 The disturbance $\alpha_{n}^{0}(\varepsilon)$ of the aberration of order $n=0,2, \cdots, 100$, due to the presence of an aberration of amplitude 1 and of the order $n^{\prime}=100$ when the system is scaled to relative size $\varepsilon=0.50$ (left) and $\varepsilon=0.98$ (right).

As an example, we consider the effect of a single high order aberration term $\alpha_{n^{\prime}}^{m}$ on the totality of $\alpha_{n}^{m}$ with $n=m, m+$ $2, \ldots, n^{\prime}$ while scaling to relative size $\varepsilon$. We take $\alpha_{n}^{m}=0$ for $n \neq n^{\prime}$ and $\alpha_{n^{\prime}}^{m}=1$, and get from Eq. (6)

$$
\alpha_{n}^{m}(\varepsilon)=\left[R_{n^{\prime}}^{n}(\varepsilon)-R_{n^{\prime}}^{n+2}(\varepsilon)\right], \quad n=m, m+2, \ldots, n^{\prime},
$$


while $\alpha_{n}^{m}(\varepsilon)=0$ when $n>n^{\prime}$. The numbers $R_{n^{\prime}}^{n}(\varepsilon)-R_{n^{\prime}}^{n+2}(\varepsilon)$ required in Eq. (7), with $n^{\prime}$ fixed and $n=m, m+2, \ldots, n^{\prime}$, can be computed simultaneously using $O\left(n^{\prime} \log n^{\prime}\right)$ operations by employing Eq. (2) in its DCT-mode. Figure 2 shows the result for $m=0$ and $n^{\prime}=100, n=0,2, \cdots, 100, \alpha_{n}^{0}(\varepsilon)$ with $\varepsilon=0.50$ and $\varepsilon=0.98$.

\section{A PROOF OF THE MAIN RESULT}

We write for integer $n, m \geq 0$ with $n-m$ even and $\geq 0$

$$
z_{n}^{m}(\nu, \mu)=Z_{n}^{m}(\rho, \vartheta)=R_{n}^{m}(\rho) \cos m \vartheta,
$$

in which the Cartesian coordinates $v, \mu$ and polar coordinates $\rho, \vartheta$ are related according to $v=\rho \cos \vartheta, \mu=\rho \sin \vartheta$ and $0 \leq$ $\rho \leq 1,0 \leq \vartheta \leq 2 \pi$. Furthermore, we let

$$
f_{n}^{m}(v)=\frac{1}{2\left(1-v^{2}\right)^{1 / 2}} \int_{-\sqrt{1-v^{2}}}^{\sqrt{1-v^{2}}} z_{n}^{m}(\nu, \mu) d \mu, \quad-1 \leq v \leq 1 .
$$

According to the formula for the Radon transform of $Z_{n}^{m}$ we have, see Ref. [10], Eq. (8.13.17),

$$
f_{n}^{m}(v)=\frac{1}{n+1} U_{n}(v), \quad-1 \leq v \leq 1,
$$

with the Chebyshev polynomial $U_{n}$ given in Eq. (3). We consider next the Zernike expansion of $f_{n}^{m}(v)$,

$$
f_{n}^{m}(v)=\sum_{n^{\prime}, m^{\prime}} \beta_{n, n^{\prime}}^{m, m^{\prime}} z_{n^{\prime}}^{m^{\prime}}(\nu, \mu)
$$

in which the $\beta^{\prime}$ s are given, due to the orthogonality ${ }^{1}$ of the $z^{\prime}$, by

$$
\beta_{n, n^{\prime}}^{m, m^{\prime}}=\frac{\left(n^{\prime}+1\right) \varepsilon_{m^{\prime}}}{\pi} \underset{v^{2}+\mu^{2} \leq 1}{\int} f_{n}^{m}(v) z_{n^{\prime}}^{m^{\prime}}(\nu, \mu) d v d \mu
$$

In Eq. (A.5) we have that $m, m^{\prime}, n, n^{\prime}$ all have the same parity and $n^{\prime} \geq m^{\prime}$, and $\varepsilon_{m^{\prime}}=1$ for $m^{\prime}=0$ and $\varepsilon_{m^{\prime}}=2$ for $m^{\prime}=$ $1,2, \ldots$ (Neumann's symbol). According to Eq. (A.3) we have

$$
\beta_{n, n^{\prime}}^{m, m^{\prime}}=\frac{\left(n^{\prime}+1\right) \varepsilon_{m^{\prime}}}{(n+1) \pi} \int_{-1}^{1} U_{n}(v)\left(\int_{-\sqrt{1-v^{2}}}^{\sqrt{1-v^{2}}} z_{n^{\prime}}^{m^{\prime}}(v, \mu) d \mu\right) d v .
$$

Then using Eqs. (A.2), (A.3) with $n^{\prime}, m^{\prime}$ instead of $n, m$, we find

$\beta_{n, n^{\prime}}^{m, m^{\prime}}=\frac{2 \varepsilon_{m^{\prime}}}{\pi(n+1)} \int_{-1}^{1} U_{n}(v) U_{n^{\prime}}(v)\left(1-v^{2}\right)^{1 / 2} d v=\frac{\varepsilon_{m^{\prime}}}{n+1} \delta_{n, n^{\prime}}$,

where $\delta$ denotes Kronecker's delta, and where we have used the orthogonality of the U's, see Ref. [11], 22.2.5 on p. 774 .

We conclude from Eqs. (A.3), (A.4), (A.7) that

$$
U_{n}(v)=\sum_{m^{\prime}} \varepsilon_{m^{\prime}} z_{n}^{m^{\prime}}(v, \mu)
$$

i.e., that

$$
U_{n}(\rho \cos \vartheta)=\sum_{m^{\prime}} \varepsilon_{m^{\prime}} R_{n}^{m^{\prime}}(\rho) \cos m^{\prime} \vartheta
$$

By orthogonality of the $\cos m^{\prime} \vartheta, \vartheta \in[0,2 \pi]$, it follows that

$$
R_{n}^{m}(\rho)=\frac{1}{2 \pi} \int_{0}^{2 \pi} U_{n}(\rho \cos \vartheta) \cos m \vartheta d \vartheta
$$

Finally, $U_{n}(\rho \cos \vartheta) \cos m \vartheta$ is a trigonometric polynomial of degree $n+m$. Therefore, the integral in Eq. (A.10) can be evaluated using the sample values of the integrand at the points $2 \pi k / N, k=0,1, \ldots, N-1$ when $N>n+m$. This yields Eq. (2).

Note. Eq. (A.10) can also be used to get accurate stationary phase approximations to $R_{n}^{m}(\rho)$ when $n$ gets large. Accordingly, $R_{n}^{m}(\rho)$ is vanishing small when $0 \leq \rho \leq \frac{m}{n}-\varepsilon$, and is oscillatory and of amplitude $O\left(n^{-1 / 2}\right)$ when $\frac{m}{n}+\varepsilon \leq \rho \leq 1-\varepsilon$ $(\varepsilon>0$ fixed, $n \rightarrow \infty)$. This can be used to explain some of the phenomena that one observes in Figure 2.

\section{ACKNOWLEDGEMENTS}

The authors thank the various referees of this paper for their valuable and constructive comments.

\section{References}

[1] M. Born and E. Wolf, Principles of Optics (7th rev. ed., Ch. 9 and App. VII, Cambridge University Press, Cambridge, MA, 2001).

[2] D. Malacara, Optical Shop Testing (2nd ed., Wiley, 1992).

[3] V. N. Mahajan, "Zernike circle polynomials and optical aberrations of systems with circular pupils" Appl. Optics. 33, 8121-8124 (1994).

[4] R. J. Noll, "Zernike polynomials and atmospheric turbulence" J. Opt. Soc. Am. 66, 207-211 (1976).

[5] See http://www.nijboerzernike.nl .

[6] A. Prata and W. V. T. Rusch, "Algorithm for computation of Zernike polynomials expansion coefficients" Appl. Optics 28, 749754 (1989).

[7] D. Calvetti, "A stochastic roundoff error analysis for the Fast Fourier Transform" Math. Comput. 56, 755-774 (1991).

[8] See http://en.wikipedia.org/wiki/Fast'Fourier transform .

[9] A. J. E. M. Janssen and P. Dirksen, "Concise formula for the Zernike coefficients of scaled pupils", J. Microlith. Microfab. Microsyst. 5, 030501 (2006).

[10] S. R. Deans, The Radon transform and some of its applications (Wiley, New York, 1983, Sec. 7.6, Eq. 6.11).

[11] M. Abramowitz and I. A. Stegun, Handbook of Mathematical Functions (Dover, New York, 1970). 